APPLICABILITY OF DUKE ACTIVITY SCALE INDEX (DASI) IN PERIOPERATIVE PREDICTION OF POSTOPERATIVE COMPLICATIONS FOR GYNAEONCOLOGY PATIENTS

${ }^{1}$ Lusine Sevinyan, ${ }^{1,2}$ Anil Tailor, ${ }^{4}$ Peter Williams, ${ }^{3}$ Pradeep Prabhu, 1,2Thumuluru Kavitha Madhuri. 'Department of Gynaecological Oncology/Royal Surrey County Hospital NHS Foundation Trust/UK; ${ }^{2}$ Faculty of Health and Medical Sciences/University of Surrey/UK; ${ }^{3}$ Department of Anaesthetics/Royal Surrey County Hospital NHS Foundation Trust/UK; ${ }^{4}$ Department of Maths and Statistics/University of Surrey/UK

\subsection{6/ijgc-2020-ESG0.233}

Introduction/Background Cohort of patients with multiple comorbidities, obesity and frailty requiring gynaeoncological interventions is continuously increasing and because of that there is an unmet need for an accurate perioperative risk prediction. The Duke Activity Scale Index (DASI) is a 12 item self-reported questionnaire based around commonly performed activities. DASI score determines functional capacity through conversion to Metabolic Equivalent of task (METs), which have been shown to indicate fitness for surgery. In our study we continue to investigate the accuracy of DASI in prediction of postoperative outcomes in the context of gynaeoncology.

Methodology A retrospective data for 290 patients was collected using a dedicated gynaeoncology database or patients' notes at a tertiary oncology centre. All of the patients had filled the DASI questionnaire prior to surgery, which we used for the analysis. Actual postoperative complications which occurred within 30 days of the surgery were also recorded. The DASI score was then compared with the occurrence of postoperative complications.

Results According to our preliminary analysis of 141 patients DASI score has not found to be a statistically significant model for prediction of postoperative complications in the general population of the gynaeoncology patients (AUC0.433). However we were able to show that a 25 point higher DASI score is predicted to deliver 1 day less in hospital. We also found that DASI score could be promising for patients with ovarian and cervical malignancy (AUC-0.634

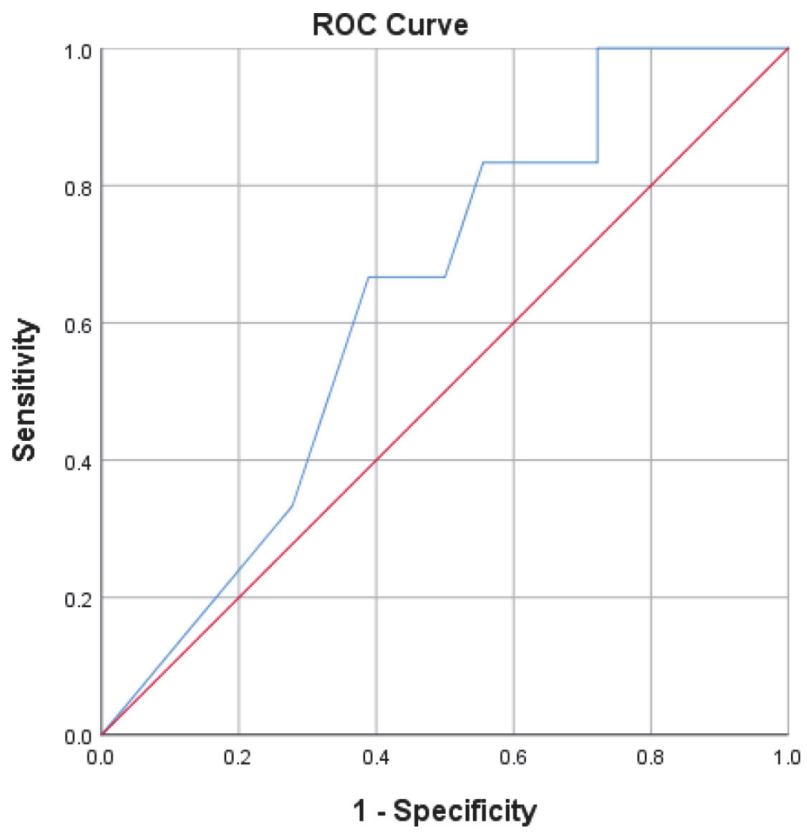

Diagonal segments are produced by ties.

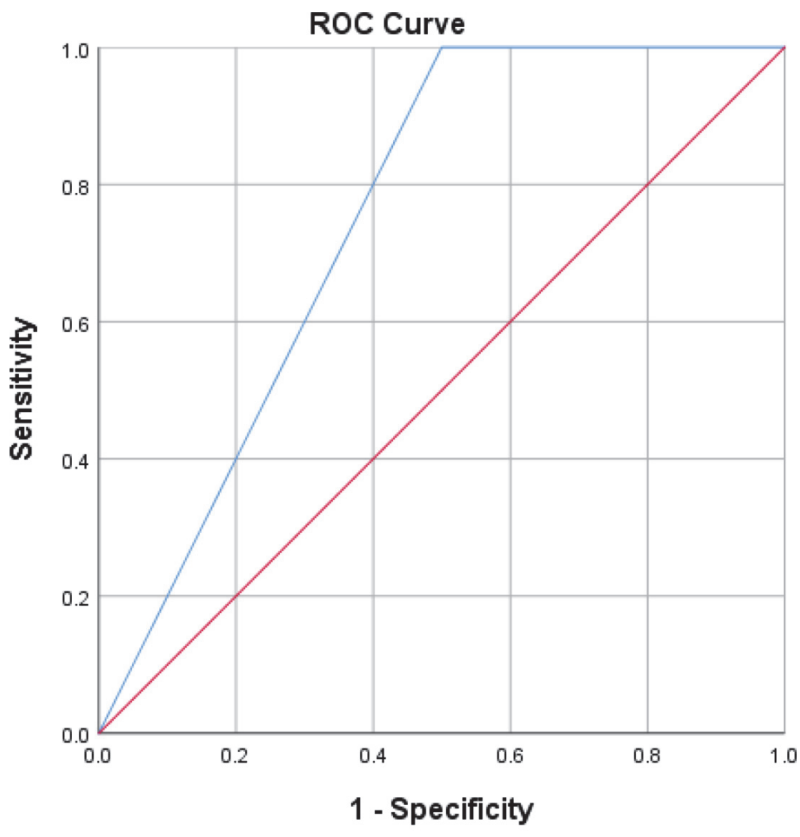

Diagonal segments are produced by ties.

Abstract 622 Figure 2

and AUC 0.750 respectively), but there were not enough patients to validate the findings in the analysed cohort (figures 1 and 2).

Conclusion DASI is an uncomplicated and straightforward tool that could be useful in perioperative estimation of postoperative complications for ovarian and cervical cancer patients. Further analysis with a larger sample size and multicentre prospective study are currently underway to validate the findings. Disclosures There are no disclosures to be made.

\section{P-POSSUM VS ACS-NSQIP: PATIENT UNDERSTANDING OF POST-OPERATIVE RISKS AND SHARED DECISION}

${ }^{1}$ Lusine Sevinyan, ${ }^{1}$ Darshana Arakkal, ${ }^{2}$ Pradeep Prabhu, ${ }^{1,3}$ Anil Tailor, ${ }^{4}$ Peter Williams,

1,3Thumuluru Kavitha Madhuri. 'Department of Gynaecological Oncology/Royal Surrey County Hospital NHS Foundation Trust/UK; ' ${ }^{2}$ Department of Anaesthetics/Royal Surrey County Hospital NHS Foundation Trust/UK; ${ }^{3}$ Faculty of Health and Medical Sciences/ University of Surrey/UK; ${ }^{4}$ Department of Maths and Statistics/University of Surrey/UK

\subsection{6/ijgc-2020-ESG0.234}

Introduction/Background Accurate postoperative surgical risk assessment is essential for surgeons and patient for assessment of potential post-operative complications especially with increasing numbers of patients with multiple comorbidities and frailty. Currently the most widely used risk scoring tool for post- operative risk prediction in the UK is P-POSSUM. Published data suggests that P-POSSUM overestimates risk causing undue patient anxiety.

We continue to explore the accuracy of the ACS-NSQIP (American College of Surgeons National Surgical Quality Improvement Program) surgical risk calculator which is a validated web-based tool based on 21 preoperative risk factors to predict 8 post-operative outcomes and compare it with $\mathrm{P}$ POSSUM.

Methodology R\&D approved retrospective study on 1200 patients undergoing robotic, laparoscopic and open surgery between 2009-2020. Data collection done through a 